\title{
Cost-effectiveness development for the postoperative care of craniotomy patients: a safe transitions pathway in neurological surgery
}

\author{
Joseph A. Osorio, MD, PhD, ${ }^{1}$ Michael M. Safaee, MD, ${ }^{1}$ Jennifer Viner, NP, ${ }^{1}$ Sujatha Sankaran, MD, ${ }^{2}$ \\ Sarah Imershein, MPH, ${ }^{3}$ Ezekiel Adigun, BS, ${ }^{4}$ Gabriela Weigel, BS, ${ }^{4}$ Mitchel S. Berger, MD, ${ }^{1}$ and \\ Michael W. McDermott, MD1
}

${ }^{1}$ Department of Neurological Surgery, ${ }^{2}$ Divsion of Hospital Medicine, ${ }^{3}$ Division of Strategic Improvement, and ${ }^{4}$ School of Medicine, University of California, San Francisco, California

\begin{abstract}
OBJECTIVE The authors' institution is in the top 5th percentile for hospital cost in the nation, and the neurointensive care unit (NICU) is one of the costliest units. The NICU is more expensive than other units because of lower staff/ patient ratio and because of the equipment necessary to monitor patient care. The cost differential between the NICU and Neuro transitional care unit (NTCU) is $\$ 1504$ per day. The goal of this study was to evaluate and to pilot a program to improve efficiency and lower cost by modifying the postoperative care of patients who have undergone a craniotomy, sending them to the NTCU as opposed to the NICU. Implementation of the pilot will expand and utilize neurosurgery beds available on the NTCU and reduce the burden on NICU beds for critically ill patient admissions.
\end{abstract}

METHODS Ten patients who underwent craniotomy to treat supratentorial brain tumors were included. Prior to implementation of the pilot, inclusion criteria were designed for patient selection. Patients included were less than 65 years of age, had no comorbid conditions requiring postoperative intensive care unit (ICU) care, had a supratentorial meningioma less than $3 \mathrm{~cm}$ in size, had no intraoperative events, had routine extubation, and underwent surgery lasting fewer than 5 hours and had blood loss less than $500 \mathrm{ml}$. The Safe Transitions Pathway (STP) was started in August 2016.

RESULTS Ten tumor patients have utilized the STP ( 5 convexity meningiomas, 2 metastatic tumors, 3 gliomas). Patients' ages ranged from 29 to 75 years (median 49 years; an exception to the age limit of 65 years was made for one 75-year-old patient). Discharge from the hospital averaged 2.2 days postoperative, with 1 discharged on postoperative day (POD) 1, 7 discharged on POD 2, 1 discharged on POD 3, and 1 discharged on POD 4. Preliminary data indicate that quality and safety for patients following the STP (moving from the operating room [OR] to the neuro transitional care unit [OR-NTCU]) are no different from those of patients following the traditional OR-NICU pathway. No patients required escalation in level of nursing care, and there were no readmissions. This group has been followed for greater than 1 month, and there were no morbidities.

CONCLUSIONS The STP is a new and efficient pathway for the postoperative care of neurosurgery patients. The STP has reduced hospital cost by $\$ 22,560$ for the first 10 patients, and there were no morbidities. Since this pilot, the authors have expanded the pathway to include other surgical cases and now routinely schedule craniotomy patients for the (OR-NTCU) pathway. The potential cost reduction in one year could reach $\$ 500,000$ if we reach our potential of 20 patients per month. https://thejns.org/doi/abs/10.3171/2018.2.FOCUS1812

KEYWORDS craniotomy; intensive care unit; cost-effectiveness; quality; brain tumor

$\mathrm{U}$ NIVERSITY of California, San Francisco (UCSF), ranks in the top 5th percentile for cost in the nation. According to our financial data for fiscal year 2015, the neurointensive care unit (NICU), which includes the neurosurgery and neurology services, is one of the most expensive of all intensive care units (ICUs) at UCSF. The majority of the direct cost can be attributed to room and board (R\&B), which amounted to $\$ 20,000,000$ for neurosurgery in fiscal year 2015 . The majority of $R \& B$ costs come from days spent in the NICU, with neurosurgery and

ABBREVIATIONS ICU = intensive care unit; NICU = neurointensive care unit; NTCU = neuro transitional care unit; OR = operating room; PACU = post-anesthesia care unit; R\&B = room and board; STP = Safe Transitions Pathway; UCSF = University of California, San Francisco.

SUBMITTED January 2, 2018. ACCEPTED February 6, 2018.

INCLUDE WHEN CITING DOI: 10.3171/2018.2.FOCUS1812. 
neurology accounting for $76 \%$ of total ICU days (Fig. 1). The NICU is significantly more expensive than other care units because of the lower staff/patient ratio and the equipment necessary to monitor vital signs and bodily functions in real time. ${ }^{3}$ In fact, the cost differential between the NICU and neuro transitional care unit (NTCU) is \$1504 per day. Reducing overutilization of the NICU promises to reduce total costs of the neurosurgery service. ${ }^{1}$

Beyond cost, potential overuse of the NICU has caused bottlenecks in the system. The bottlenecks occur when there are patients who are requested to transfer out of NICU, but there are no lower-acuity beds available, and therefore these patients remain in the NICU until a bed becomes available. ${ }^{2}$ This subpopulation of patients who are expected to have a short stay in the NICU for observation and monitoring but whose stays will be longer than required is not only a contributor to a bottleneck within the system, but it could also be a contributor to the higher costs. If, however, the increased R\&B cost for the NICU could be exchanged for the cost of the NTCU, potential saving could be achieved. Also, at our institution, when the NICU is at full capacity, scheduled elective craniotomy cases cannot proceed or are placed on a hold until a NICU bed becomes available. ${ }^{2}$

Traditionally, all patients undergoing a neurosurgical craniotomy to treat tumors coming from the operating room (OR) are transferred to the NICU for hourly neurological examinations to identify any postoperative complications as early as possible. ${ }^{3,4}$ These same patients, often the following morning (i.e., in the NICU for less than 24 hours since surgery), transition to lower-acuity units that also have less frequent monitoring. Our providers predict, however, that certain patient subpopulations with expected very low complication and adverse event profiles may not require hourly monitoring with the higher level of care provided in the NICU after surgery, and there would potentially be no difference in adverse events after surgery if they were managed in the NTCU with slightly less frequent monitoring. These patients, if identified appropriately, would be served equally well by moving from the OR to the post-anesthesia care unit (PACU) to the NTCU (Fig. 2). ${ }^{5}$

The population first identified as the best candidates to bypass the NICU were overall healthy patients with simple supratentorial tumors that were small in size, and they were selected by our senior author (M.W.M.). These patients are stable, have low-acuity needs, and are at minimal risk for poor neurological outcomes. Table 1 summarizes the inclusion criteria. When performing a retrospec-

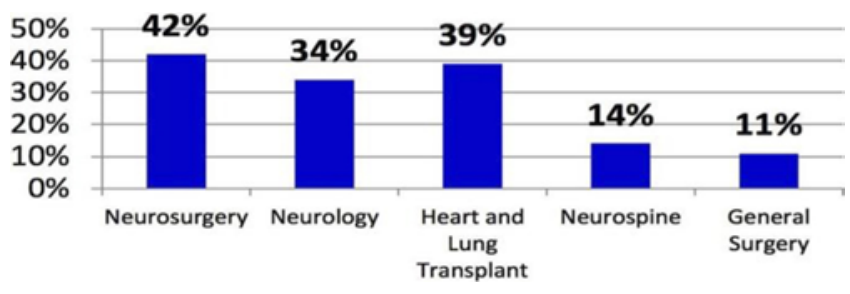

FIG. 1. The percentage of time spent in the NICU by service is shown. Neurosurgery and neurology account for $42 \%$ and $34 \%$, respectively, of total ICU days.

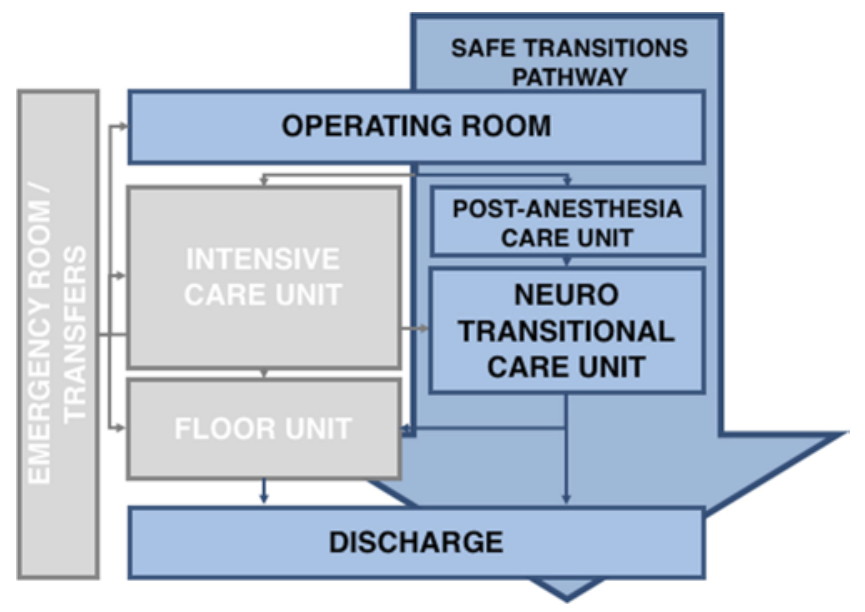

FIG. 2. Flow diagram showing how the locations that the traditional pathway (gray), and the safe transitions pathway (blue) interact.

tive review of supratentorial tumors treated at our institution, we found approximately 5-20 patients per month in this identified subpopulation who would normally go to the NICU but who might instead be able to go to the PACU and NTCU. Having these patients bypass the NICU was anticipated to result in lower costs for $\mathrm{R} \& \mathrm{~B}$, decrease bottlenecking in the NICU, and increase the potential for an improved patient experience. A benefit to this pathway was that it could be generalized to other neurosurgical care patients, not just those with supratentorial tumors, and have a greater impact on cost savings.

\section{Methods}

\section{Target}

We had several main goals for this project. 1) One was to increase the number and type of patients utilizing the Safe Transitions Pathway (STP) by continuing to recruit patients from identified target populations. In addition, we sought to identify other populations that are eligible and that would also be expected to have no difference in adverse events. 2) Another goal was to document in a pilot study that this proposed pathway was safe and there were no adverse patient events related to the PACU and NTCU pathway. 3) A third goal was to analyze financial savings. 4) Our final goal was to use data to support the STP such that it could be expanded for use by other surgeons and for other surgical procedures.

TABLE 1. Eligibility criteria for inclusion into the safe transitions pathway

\begin{tabular}{lll}
\hline \multicolumn{1}{c}{$\begin{array}{c}\text { Preoperative } \\
\text { Criteria }\end{array}$} & \multicolumn{1}{c}{$\begin{array}{c}\text { Intraoperative } \\
\text { Criteria }\end{array}$} & $\begin{array}{c}\text { Current } \\
\text { Cases }\end{array}$ \\
\hline Routine craniotomy & Routine extubation & Meningioma \\
\hline Supratentorial location & No intraoperative events & Glioma \\
\hline Size $<3 \mathrm{~cm}$ & Operative time $<5$ hours & \\
\hline Age $<65$ yrs & Blood loss $<500 \mathrm{ml}$ & \\
\hline $\begin{array}{l}\text { No comorbidities requiring } \\
\text { ICU (cardiac or pulmonary) }\end{array}$ & \\
\hline
\end{tabular}


The reduction in cost, achieved by reducing extra NICU days, translates into multifaceted savings. In just the identified subpopulations, using inclusion criteria for the pilot study, 12 patients per month following the STP, which results in a mean of 1.5 fewer NICU days per patient, equates to nearly $\$ 400,000$ in savings per year, almost $0.5 \%$ of direct costs. When reviewing our retrospective data and evaluating how many patients would potentially be captured into the STP, we noted that the median was 12 patients/month (range 5-20 patients/month), and therefore we know that 12 patients per month is attainable within our identified populations. There are also a number of other types neurosurgery patients who use the NICU in a similar way for frequent neurological examinations, often only until the morning after surgery (i.e., they are in the NICU less than 24 hours since surgery), who could also transition to lower-acuity units with less frequent monitoring. These include patients who have undergone Chiari malformation decompressions, microvascular decompressions for trigeminal neuralgia, and surgery for infratentorial tumors and other types of supratentorial tumors. These patients were similarly identified, as our initial population, by looking at trends for postoperative ICU stay and having similar indications for the ICU needs (frequent monitoring). Table 2 shows the STP and expanded diagnoses/procedures that would later be added into the pathway. Table 3 demonstrates cost-reduction estimates based on the mean number of patients included in the STP driven by our retrospective calculation. The maximum number of patients captured would include all of the expanded diagnoses/procedures.

Although the STP focuses most directly on lowering cost, we also envision that STP will achieve 2 other goals: 1) improve the patient experience and 2) create an optimal work experience for our nursing staff, both in the PACU and NTCU. In bypassing the NICU, patients will have a more comfortable stay in the hospital, with family able to accompany patients overnight in their rooms and also be more involved in direct care and observation of their loved ones. The reduced NICU workload and subsequent relief of bottlenecking will improve the elective surgery patient/ provider experience by allowing procedures to proceed on the scheduled days as originally booked. We anticipate as the project continues and we generate safety, efficiency, and cost-savings results, the current culture will shift and similar STPs will spread to other surgical services and contribute to our institution's commitment to discovery and advancement of knowledge.

\section{Gaps}

Root cause analysis reveals unit culture as a contributing factor in maintaining the costly system that has had all postoperative craniotomy patients sent to the NICU, without carefully identifying those who could have been managed appropriately in lower-acuity settings. Until piloting the STP, the mindset among the neurosurgery unit staff was that patients "going to the NICU is the way it has always been done." A culture of delivering the highestacuity care prevails, and few benefits are perceived in being cared for after surgery in lower-acuity settings. Neurosurgeons see hourly neurological checks as assurance for
TABLE 2. Pilot study population and future expansion to other populations

\begin{tabular}{cl}
\hline \multicolumn{1}{c}{ Pilot Study } & \multicolumn{1}{c}{ Future Program Expansion } \\
\hline Supratentorial tumors & Supratentorial tumors \\
\hline & Infratentorial tumors \\
\hline & Chiari malformation decompression \\
\hline & Microvascular decompression \\
\hline
\end{tabular}

the safety of their patients, and they may perceive cost savings as inadequate justification for the risk of catastrophic neurological events. Despite this prevailing culture, we noted that this pathway of OR-NICU has not always been the case and that, historically, low-risk patients did indeed transfer directly from the OR to the PACU or NTCU. Also, there has not been an increase in adverse events; on the contrary, for the population we chose to follow the STP, adverse events have been rare. This discovery would appear to support a pathway that diverts certain postoperative patients away from the NICU.

Among surgical and nursing providers, the STP aims to enable a change in core behavior away from the default transfer of patients to the NICU when the patients are clinically eligible for the NTCU. Part of this change depends on proving that STP patients receive noninferior, if not improved, safety and quality of care as patients who follow the traditional pathway. To date, the STP seems to have reduced the number of blood draws and tests, but this requires further analysis. The absence of an arterial line in the NTCU makes access to blood samples another intervention, which prompts decision making as to whether the test is necessary or not. Prior to the STP pilot, there had been no documented demonstration of how to safely or efficaciously provide a pathway to implementing change of neurosurgical care for cost reduction; however, initial pilot data seems promising in this regard.

\section{Intervention}

Current Workflow of Interventions in Place

1. The patient is evaluated by the surgeon and craniotomy is recommended as the treatment modality.

2. The surgeon places the surgery order and postoperative destination in the electronic medical record.

3. The nurse practitioner (NP) working with the surgeon identifies and suggests the STP if criteria are met.

TABLE 3. Cost reduction prediction based on amount of patients that would be included into the safe transitions pathway

\begin{tabular}{lll}
\hline Monthly Patients & Yearly Patients & Annual Cost Savings* \\
\hline 5 patients $/ \mathrm{mo}$ & 60 patients $/ \mathrm{yr}$ & $\$ 135,000 / \mathrm{yr}$ \\
\hline 8 patients $/ \mathrm{mo}$ & 96 patients/yr & $\$ 216,000 / \mathrm{yr}$ \\
\hline 12 patients $/ \mathrm{mo}$ & 144 patients/yr & $\$ 324,000 / \mathrm{yr}$ \\
\hline 20 patients $/ \mathrm{mo} \dagger$ & 240 patients/yr & $\$ 540,000 / \mathrm{yr}$ \\
\hline * Estimates include savings of $\$ 2250$ per patient calculated from a mean of \\
1.5 ICU days. \\
$\begin{array}{l} \\
\text { diagno 20-patients-per-month figure includes future program expansion of }\end{array}$
\end{tabular}

Neurosurg Focus Volume $44 \cdot$ May 2018 

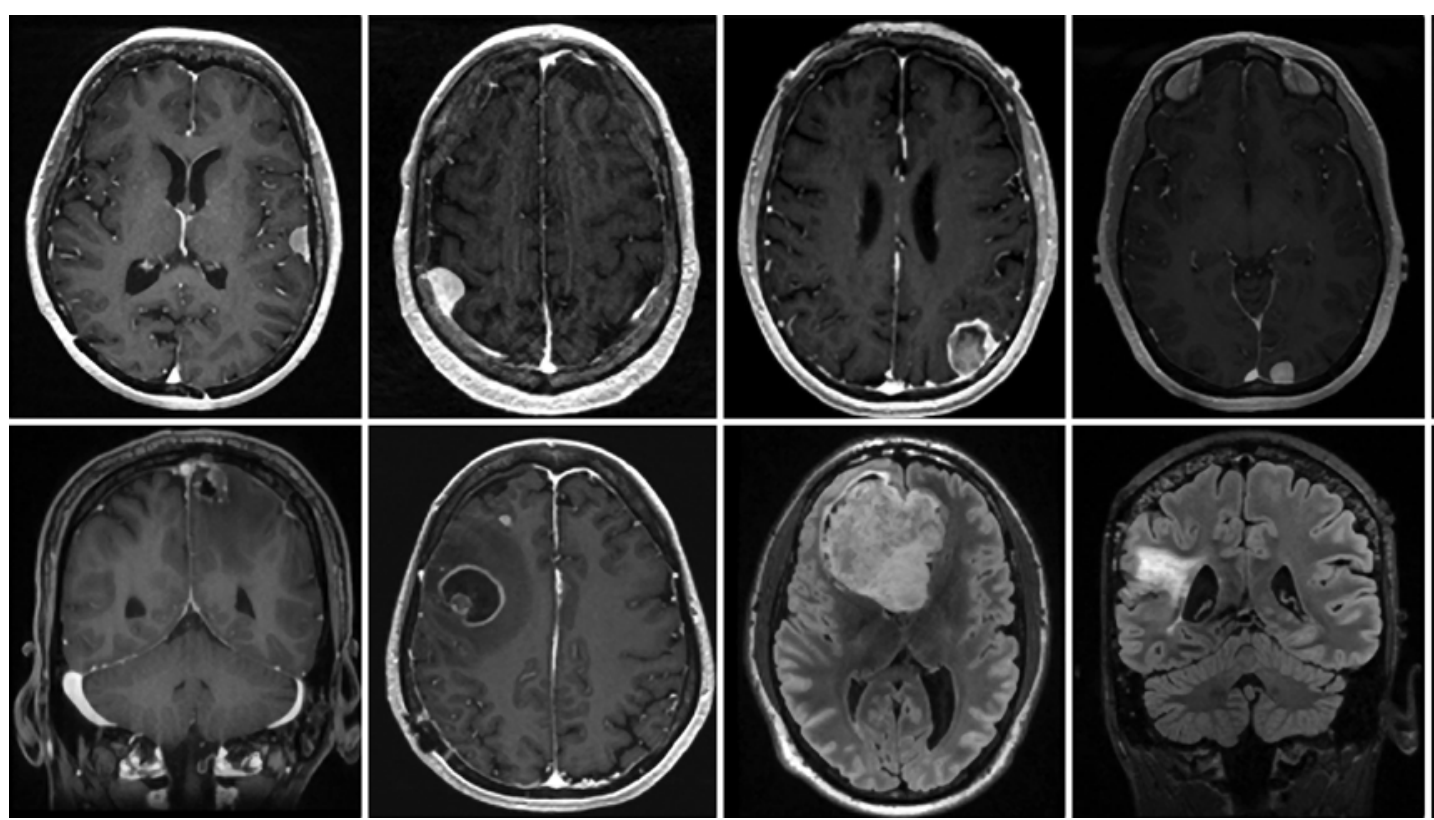

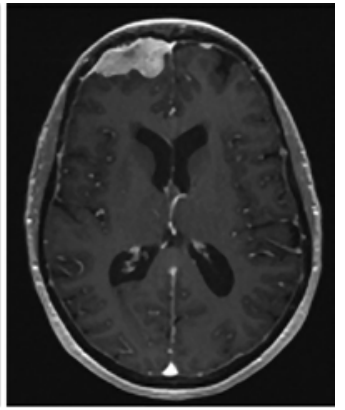

FIG. 3. Images obtained in the first 10 patients to complete the STP. Shown are a combination of axial and coronal representative slices that capture the tumor that was the target for resection.

4. The NP meets weekly with the practice coordinator and identifies eligible STP cases. If patients are identified, the NP confirms with the surgeon, and, if appropriate, the postoperative destination on the surgical order is changed to NTCU.

5. The NP discusses the plan for STP with patient (on the phone or in the clinic) and provides an informative pamphlet.

6. The week prior to STP admission, the NP sends an email to the following: RN for bed control, PACU management, NTCU manager and charge nurses, chief and service residents, hospitalist, and neurosurgeon.

7. The day of surgery, the NP reminds the surgeon of the STP plan and the need for the surgeon to review the plan with the anesthesiologist. The NP calls NTCU to reserve a monitored bed; rounds are made in the PACU and NTCU for support.

\section{Results}

Ten tumor patients were safely managed using the STP (5 with convexity meningiomas, 2 with metastatic tumors, and 3 with gliomas) (Fig. 3). Patients ranged in age from 29 to 75 years (mean 49 years; an exception to the age limit of 65 years was made for one 75-year-old patient). They were discharged on average 2.2 days after surgery, with one discharged on postoperative day (POD) 1, 7 on POD 2, 1 on POD 3, and 1 on POD 4. Of the 10 patients, none has required escalation in the level of nursing care and none has been readmitted. Additionally, no patient visited the emergency department within 14 days of discharge; only 1 visited the emergency department within 30 days of discharge (for a seizure on POD 15). For the 10 patients, the STP reduced hospital cost by $\$ 22,560$, and there were no morbidities. These preliminary data from the pilot indicate that STP is not inferior to the traditional OR-NICU-floor transition in terms of quality and safety.

While not measured to date, patient experience, we anticipate, may improve with the STP compared with that of traditional care. There is evidence that patients who experience fewer transitions during hospitalization have higher satisfaction scores, and the STP-treated patients may express more satisfaction with their care if they bypass the NICU. In addition, NICU patients consistently complain of difficulty sleeping and lack of family presence as barriers to recovery, and these factors may be alleviated by adoption of the STP.

\section{Discussion}

The Safe Transitions Pathway that was developed was successful in implementation, it demonstrated reduced cost, and the patients who completed the pathway did not exhibit any adverse events. Adverse outcomes that were being evaluated were return to the OR, neurological decline, death, length of stay, and readmission. There were no adverse events that were noted in the first 10 patients that completed the pathway.

When developing the pathway there was a careful evaluation of the various steps in the pathway that could impact cost. One particular added cost was that which was associated with the additional time spent in the ICU, when there were no lower-acuity beds available, and the patient was kept in the ICU until a bed became available. At our institution, ICU charges cannot be adjusted to a different level of acuity cost when a patient is physically in a critical care unit; patients are charged an ICU rate if they are boarded in the ICU. Additionally, the nursing staff remains critical care nurses because they are trained and staffed according to the acuity level. The hospital units are 
segregated by acuity, and nursing staff is designated based on unit and level of acuity. Although lowering charges for these patients would have an impact on cost, our institution has a billing infrastructure and staffing that would not allow for these changes to be made.

Expansion to other patient populations was implemented sequentially, and iterations to the current inclusion criteria for the STP have also been made in a similar way. The process, as it has expanded, has created a foundation of STP patients who have completed the pathway without adverse events, which allows us to gain further support to engage additional providers to include and expand the current population of patients. During the implementation phase, although other target patient populations were identified, not all providers were initially supportive, but further evidence provided the reassurance that was needed to gain the confidence to enroll and expand the current cohort. This tactic worked well when strategizing how to implement growth after the initial success of the pathway was achieved.

There are 3 factors that will drive the sustainability of the STP. First, establishment of electronic medical record protocols in using order sets will provide the basis for sustainability of the pathway. Second, expansion of the target population to multiple surgical criteria and multiple neurosurgeons will help solidify the pathway as appropriate for multiple populations of patients. Third, continued establishment of evidence that patients enrolled in the pathway have similar or better outcomes than patients who receive traditional care and the channeling of these safety data back to providers will help promote and sustain the pathway in the long-term.

As the pathway continues to grow and expand, there are ongoing evaluations focused on identifying adverse events. There is also a protocol in place for the evaluation of an adverse event, should one occur, that would allow for all persons involved to conduct a critical evaluation if an adverse event could have been prevented outside of the pathway and if the event was addressed within the pathway in an appropriate way.

\section{Conclusions}

The STP is a new and efficient pathway for the postoperative care of neurosurgery patients. It has reduced hospital cost by $\$ 22,560$ for the first 10 patients, and there were no morbidities. Since this pilot, we have expanded the pathway to other surgical cases and now routinely schedule craniotomy patients for an OR-NTCU transition. The potential cost reduction in 1 year could reach $\$ 500,000$ if we reach our potential of 20 patients per month.

\section{References}

1. Beauregard CL, Friedman WA: Routine use of postoperative ICU care for elective craniotomy: a cost-benefit analysis. Surg Neurol 60:483-489, 2003

2. Bui JQ, Mendis RL, van Gelder JM, Sheridan MM, Wright $\mathrm{KM}$, Jaeger M: Is postoperative intensive care unit admission a prerequisite for elective craniotomy? J Neurosurg 115:1236-1241, 2011

3. Kelly DF: Neurosurgical postoperative care. Neurosurg Clin N Am 5:789-810, 1994

4. Knaus WA, Draper E, Lawrence DE, Wagner DP, Zimmerman JE: Neurosurgical admissions to the intensive care unit: intensive monitoring versus intensive therapy. Neurosurgery 8:438-442, 1981

5. Taylor WA, Thomas NW, Wellings JA, Bell BA: Timing of postoperative intracranial hematoma development and implications for the best use of neurosurgical intensive care. $\mathbf{J}$ Neurosurg 82:48-50, 1995

\section{Disclosures}

The authors report no conflict of interest concerning the materials or methods used in this study or the findings specified in this paper.

\section{Author Contributions}

Conception and design: Osorio, Safaee, Viner, Berger, McDermott. Acquisition of data: Osorio, Safaee, Viner, Imershein, Adigun, Weigel, McDermott. Analysis and interpretation of data: all authors. Drafting the article: Osorio, Safaee, Viner, Sankaran, Berger. Critically revising the article: Osorio, Imershein, McDermott. Reviewed submitted version of manuscript: Osorio, Safaee, Viner, Imershein, Adigun, Weigel, Berger, McDermott. Approved the final version of the manuscript on behalf of all authors: Osorio. Statistical analysis: Osorio, Safaee. Administrative/technical/ material support: Osorio, Safaee. Study supervision: Berger, McDermott.

\section{Correspondence}

Joseph A. Osorio: University of California, San Francisco, CA. osorioj@neurosurg.ucsf.edu. 